\title{
Actitudes de la ciudadania hacia la separación de residuos en Coatepec, Veracruz, Mexico
}

\author{
Citizens attitudes towards waste separation in \\ Coatepec, Veracruz, Mexico
}

\author{
Astrid Wojtarowski Leal \\ María de los Ángeles Piñar Álvarez \\ José Luis MaRín MuñIZ \\ Universidad Pública Colegio de Veracruz (COLVER) \\ astrid_leal@yahoo.com.mx (MÉXICO)
}

Recibido: 23.032018

Aceptado: 20.02.2019

\section{RESUMEN}

En el presente documento, se presentan los resultados de un estudio en Coatepec, a partir de los antecedentes de una investigación en Teocelo; ambos municipios enclavados en la Sierra Madre Oriental veracruzana. El primero con una fuerte problemática de gestión de residuos sólidos urbanos (RSU); el último, en contraste, ha sido un caso exitoso de gestión integral desde el año 2001.

El objetivo del estudio fue examinar las actitudes de habitantes de Coatepec sobre el cambio en el comportamiento ambiental relativo a políticas públicas, en particular a la separación de residuos, así como recabar sus opiniones sobre la calidad de la gestión municipal en materia de limpia pública.

Metodológicamente, se aplicó una escala de Likert a 108 individuos, con preguntas asociadas a la actitud en sus componentes conativo, cognitivo y afectivo.

Los resultados muestran una alta disposición de los encuestados para separar los residuos y participar en el proceso de transformación. Asimismo, una opinión desfavorable sobre el funcionamiento del área de limpia pública en Coatepec. Finalmente, persiste un marcado interés de la ciudadanía porque en su municipio se ponga en marcha un programa de gestión integral de residuos sólidos urbanos semejante al que aplica el municipio vecino de Teocelo.

Se concluye con la importancia de que habitantes y equipo de gobierno colaboren en materia de limpia pública. La ciudadanía toma acciones de separación o muestra intenciones de hacerlo y tiene conocimiento sobre el tema de residuos en su primer nivel de separación (orgánico e inorgánico). El análisis sobre ac- 
titud realizado en sus dimensiones conativa, cognitiva y afectiva, señala lo que falta por atender en la población con respecto a la separación y buen manejo de los residuos sólidos desde el nivel individual, colectivo y de los representantes políticos, línea base para el diseño de estrategias de planeación estratégica y de educación ambiental.

\section{PALABRAS CLAVE}

Gestión integral de residuos sólidos urbanos, desarrollo sustentable, Municipio de Teocelo, Municipio de Coatepec, México.

\section{ABSTRACT}

The results of a study carried out in Coatepec are presented in this study, which were based on a previous research conducted in Teocelo. Both municipalities are located in the Sierra Madre Oriental, Veracruz. The Coatepec research had complications related to the management of solid urban waste. However, the Teocelo study has been a successful case of integral management since 2001 .

The main goal of the study was to examine the attitudes of Coatepec residents about the change in environmental behavior related to public policies, in particular the separation of waste, as well as to gather their opinions on the quality of municipal management in terms of public cleanliness.

In the methodologyl, a Likert scale was applied to 108 individuals, with questions associated to attitudes in their conative, cognitive and affective components.

Findings showed that participants are highly motivated to separate waste and contribute in the transformation process. Furthermore, they expressed a negative opinion on street cleaning in Coatepec. Finally, citizens strong interest on establishing a program of integral management of urban solid waste in their municipality persists. They want a similar one to that applied on Teocelo.

In conclusion, it is vital for inhabitants and the government to team up and work together in matters of public cleanliness. Citizenship takes action in waste separation or shows intentions to do so, being aware of the waste issues in its first level of separation (organic and inorganic). The attitudes analysis, conducted in its conative, cognitive and affective dimensions, points out what is missing in the population regarding waste separation and good management of solid waste from the individual, collective and political representatives level. This is the baseline towards designing strategies for strategic planning and environmental education. 


\section{KEY WORDS}

Integrated management of urban solid waste, sustainable development, $\mathrm{Mu}-$ nicipality of Teocelo, Municipality of Coatepec, Mexico.

\section{INTRODUCCIÓN}

La idea de que el futuro, en términos de la translimitación en el uso de los recursos, ya nos alcanzó, fue planteada por Ernest García en 2005. Esta expresión, crudamente realista, podría servir tanto para la inacción como para la búsqueda de alternativas que, si bien se antojan de grandes dimensiones y a nivel global, en los debates sobre el tema se sugiere dar relevancia y prioridad al trabajo local.

Es así como la gobernanza y teorías de desarrollo local alternativo se despliegan en la búsqueda de que la problemática ambiental no genere comportamientos socioambientales caóticos (García, 2006). En este intento, ha sido fundamental la consideración de que la sociedad y el medio ambiente no debían seguirse comprendiendo como entes separados sino como un sistema socio-ecológico (Berkes et. al, 2003). Para García (2006) la cuestión clave en este sistema no radica en mantenerlo bajo control sino en lograr que sea flexible. Bajo esta perspectiva, la idea de desarrollo sustentable se acercaría a un proceso de acción consciente en la búsqueda de estados deseables y donde las condiciones locales se tomarían en cuenta, primando sobre metas y fórmulas homogeneizantes.

Según apunta García (2006), estas vías flexibles o alternativas de desarrollo centran su discurso en diferentes nodos: la re-localización (Mander y Goldsmith, 1996), la diversidad cultural (Escobar, 1994), o el post-desarrollo (Sachs y Esteva, 1996), pero todas coinciden en que la escala local-regional es la más adecuada tanto para la resistencia a un desarrollo homogeneizante como para la construcción de alternativas a los problemas asociados al uso excesivo de los recursos.

Aunque esta problemática es mucho más extensa, aquí pondremos atención en uno de los problemas más visibles a nivel local, que es la generación constante de residuos. La situación tiene, en otros orígenes, la ideología y la práctica del consumismo, lo cual implica que las soluciones serán complejas y que deberán trascender a la pura gestión. Sin embargo, desde la perspectiva municipal, el mal manejo desde la fuente se convierte en un enorme reto, además de los riesgos asociados a la salud ambiental y humana. Por ello la relevancia de la separación adecuada desde la fuente, así como de un manejo municipal que sea consecuente con las acciones de los ciudadanos. La gestión adecuada se hace cada vez más urgente ante las estimaciones sobre la producción de basura a nivel global, que muestran que para el 2025 se incrementará en $70 \%$ los residuos generados en las ciudades (Banco Mundial, 2012).

El problema de los residuos ha sido escrutado desde diversos ángulos: desde la perspectiva propiamente técnica de la producción, manejo y destino final; desde el enfoque de la psicología cognitiva y la sociología política, ambos a través 
de la Teoría del Comportamiento Planificado (TCP) (Fishben y Ajzen, 1973). Desde la psicología cognitiva los estudios han explorado comportamientos individuales (Tylor y Todd, 1995; Chu y Chiu, 2003; Knussen et al., 2004 y Durán et al., 2009) y en el caso de la sociología política, se añaden elementos teóricos de la identidad social, para exponer el fenómeno de las actitudes trascendiendo el enfoque individual y encontrar los factores colectivos que inciden en la participación ciudadana en el tema de la gestión de residuos (Wojtarowski et al., 2018).

Los trabajos de psicología cognitiva, en la búsqueda por definir cuál de los componentes asociados al comportamiento (actitud, norma social y control percibido) tienen mayor peso, han arrojado una variedad de resultados que impide aclarar esta interrogante. Tylor y Todd (1995) y Durán et. al., (2009), encontraron que la variable más significativa para explicar los comportamientos es el control percibido, mientras que Boldero (1995) y Knussen et al., (2004), reportaron que esa misma variable resultaba poco significativa. Por su parte, la revisión realizada por Ajzen (1991), de estudios que relacionan actitud con comportamiento, encontró que en algunas ocasiones se mostraba una correspondencia significativa y en otras solamente parcial.

De lo anteriormente planteado, aunado a los resultados de un estudio basado en la TCP, que incorpora elementos de identidad social y un enfoque colectivo (Wojtarowski et. al., 2018), se deduce que la actitud, la norma social y el control percibido se relacionan de manera compleja con la toma de decisiones y el comportamiento, y que resulta aventurado determinar cuál de ellos tiene un peso mayor. Bajo esta perspectiva el comportamiento se explicaría en la relación compleja de aquellos elementos, en cuyo interior encontramos a su vez, componentes cognitivos, contextuales y emocionales. Tales componentes se encuentran asociados a los que conforman la actitud según Sabater (1989) y que serán revisados más adelante. De manera que puede plantearse que es relevante indagar en dichos componentes, dado que esto permitiría obtener pistas sobre los probables comportamientos ambientales.

\subsection{Los residuos sólidos municipales, problema de municipios mexicanos}

La disposición y gestión inadecuada de residuos genera graves afectaciones ambientales y de salud pública (Acurio et al., 1997; Lagrega y Evans, 1996; Nagao et al., 2003; Soumaré et al., 2003), de manera que su atención adecuada debe ser tema prioritario en el diseño y sobre todo en la implementación de políticas públicas. El Banco Mundial (2012), advierte que la gestión de residuos será una de las labores más importantes que deberá brindar un municipio y sugiere trabajar en su fortalecimiento.

En México, las políticas ambientales en materia de residuos sólidos urbanos (RSU) o residuos municipales, siguen siendo un tema desdeñado a nivel nacional (Piñar-Álvarez, 2014) y, aunque se muestran mejoras puntuales a nivel de estados y de municipios tras la aplicación de 'políticas públicas federales y estatales para el fortalecimiento de numerosos municipios de México (Lizama et 
al., 2016; Lizama, 2018), el problema sigue latente en las generaciones presentes de gobiernos locales.

Hay pocos municipios que llevan a cabo una política exitosa en relación a la planeación y manejo integral de los residuos sólidos urbanos, uno de ellos es Teocelo, ubicado en la zona central montañosa, Corredor Ecológico de la Sierra Madre Oriental del estado de Veracruz, mismo que se ha caracterizado por ser pionero en la gestión integral de residuos sólidos urbanos (Medina y Castillo, 2014). En el año 2002, Teocelo recibió el premio Gobierno y Gestión Local por este programa, convirtiéndose en un referente nacional en el tema: su experiencia puede ser útil para iniciar a otros municipios y regiones en una labor similar. Esta premisa guió el trabajo de investigación cuyos primeros resultados aquí se presentan.

Tomando como base la indagación sobre los factores que incidieron en el éxito del programa en Teocelo (Wojtarowski et al., 2018), la propuesta fue iniciar a este municipio como escuela de otro con características similares. Para ello se eligió a Coatepec, un municipio más grande pero similar a Teocelo desde el punto de vista geográfico, cultural y productivo. Aquí se mostrará la primera parte de este proceso, que consistió en evaluar las actitudes de habitantes de Coatepec sobre el cambio en el comportamiento ambiental relativo a políticas públicas, en particular a la separación de residuos.

\subsection{Teocelo y Coatepec: parte de un mismo territorio.}

Coatepec y Teocelo se asientan mayormente en un ecosistema denominado "bosque de niebla", en las estribaciones del Cofre de Perote o Nahucampatepetl, un volcán masivo que se levanta 4282 metros sobre el nivel del mar. Esta zona tiene características particulares pues en ella las nubes se condensan y provocan abundantes lluvias, humedad y niebla gran parte del año. Los bosques de niebla, han sido motivo de interés para ecólogos y conservacionistas desde la década de los noventa (Williams, 2007), debido a la destrucción a que se han sometido a nivel mundial y a la importancia de su conservación por la enorme biodiversidad que albergan, así como los servicios ambientales ${ }^{1}$ que brindan, como son: provisión y captura de agua, estabilización del régimen hídrico, ecoturismo, recreación (Cadena et al., 2007) y cuidado de suelos contra la erosión (Manson, 2004). Ambos municipios comparten también el origen cultural, derivado de la cultura totonaca (INAFED, 1998) y el mestizaje étnico y cultural de la zona, el cual se conformó a partir de la fusión entre los pueblos originarios y los conquistadores europeos.

${ }^{1}$ Según Daily (1997), los servicios ambientales son los procesos a partir de los cuales los ecosistemas naturales y las especies que en ellos se encuentran sustentan a los seres humanos. 

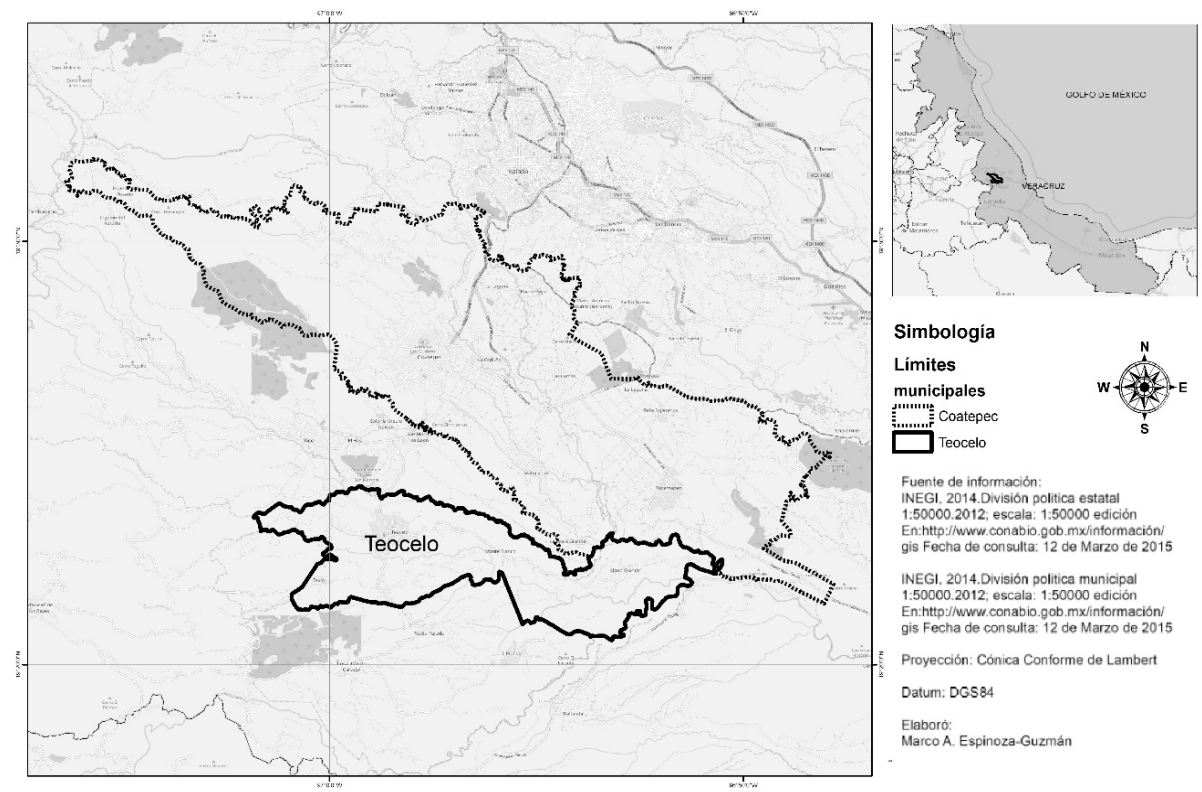

Fuente de información:

INEGI, 2014. División politica estatal
$1-50000.2012$; escala: 1,50000 edicio

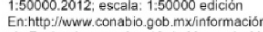

gis Fecha de consulta: 12 de Marzo de 2015

INEGI, 2014.División politica municipal

1.50ctp:/wwwiconabio 1.50000 edición

gis Fecha de consulta: 12 de Marzo de 201

Proyección: Cónica Conforme de Lambert

Datum: DGS84

Elaboro:

Marco A. Espinoza-Guzmán

Figura 1. Región de estudio, Teocelo y Coatepec en Veracruz, México

Fuente: Elaboración propia (2018)

Teocelo, cuenta con 17369 habitantes, 8837 mujeres y 8532 hombres. El municipio ha tenido una histórica vocación rural, pero actualmente el sector terciario es el de mayor crecimiento: de la población ocupada, $49.2 \%$ se dedica al comercio o los servicios, quedando una distribución idéntica para los sectores primario y secundario, los cuales ocupan $23.9 \%$ cada uno, de la población económicamente activa.

El cultivo de café es la actividad productiva fundamental, la que aporta más ganancias dentro del sector primario, seguido por la caña de azúcar y la macadamia. Su clima es semicálido húmedo con lluvias todo el año, con un rango de temperatura de 18-24 grados centígrados, sus niveles de altitud se encuentran entre los 500 y los 1400 msnm (SEFIPLAN, 2015 a).

Por su parte, Coatepec colinda al sur con Teocelo y una parte de su territorio (35\%) muestra un mismo clima; sin embargo, la mayor parte del municipio coatepecano (42\%) posee un clima semicálido húmedo con abundantes lluvias en verano. El rango de temperatura para Coatepec es de 10-24 grados centígrados y tiene una altitud media de $1200 \mathrm{msnm}$. Su población es de 92,848 habitantes, de los cuales, 48200 son mujeres y 44648 son hombres. De su Población Económicamente Activa ocupada, 65\% se dedica al sector terciario, $25.3 \%$ al secundario y $9.4 \%$ al primario; con ello, se observa el mismo fenómeno de tercerización de la economía que en Teocelo; pero a diferencia de este último, Coatepec tiene 
un porcentaje significativamente menor de población ocupada en el sector primario. Como en el caso de Teocelo, la mayor superficie cultivada corresponde al café, sin embargo, en Coatepec el cultivo que presenta un mayor volumen de producción y ganancias es la caña de azúcar, seguido por el café y la macadamia, respectivamente (SEFIPLAN, 2015b).

\subsection{Antecedentes en el municipio de Teocelo}

Los problemas derivados del inadecuado manejo de RSU van desde el deterioro del suelo por los lixiviados a la contaminación visual, del agua y aire, hasta incidencias negativas en la salud de la población que se encuentra expuesta a los desechos (Troschinetz y Mihelcic, 2009).

Una estrategia que se sugiere para minimizar tales problemáticas, consiste en la activación de programas de gestión o manejo integral de RSU, con los objetivos de reducir, reciclar y reutilizarlos. Sin embargo, en ese sentido, se sabe que no todos los esfuerzos han sido exitosos, pues depende de la voluntad de los ayuntamientos y de las actitudes y valoraciones que los ciudadanos tienen sobre el fenómeno, aunado a su disposición para participar (Armijo et al. 2012; Wojtarowski et al. 2018).

A diferencia de otros municipios, el municipio de Teocelo ha llevado a cabo un programa de gestión que ha resultado exitoso. La investigación que dio cuenta de los factores de tal fenómeno, concluyó que el comportamiento y la disposición para participar en el programa por parte de los ciudadanos de este municipio se explican a través de la integración de elementos cognitivos, contextuales y emocionales. Si los individuos perciben que la acción es fácil de llevarse a cabo, es positiva y que existen las condiciones para la continuidad por parte de la autoridad municipal en las etapas posteriores a la acción ciudadana, tendrán una mejor disposición para participar. El caso del componente emocional o afectivo "implica una evaluación modélica de los valores y aspiraciones de la colectividad a la que se pertenece y de sus proyectos sociales sucesivos" (Wojtarowski et al. 2018:28).

En Teocelo, la separación transitó de un acto que inició a petición de la autoridad municipal a una acción de autonomía ciudadana. Los habitantes de la localidad exigieron la restitución del programa después de un lapso en que fue suspendido; esto fue posible porque el hábito ya se encontraba profundamente arraigado y los ciudadanos no concebían otro tipo de comportamiento, pues percibían que desechar todos los residuos juntos era una muestra de retroceso (Wojtarowski et al., 2018). 


\subsection{Los componentes de la actitud}

Desde la perspectiva de Fernández (1982), la actitud es un estado de disposición psicológica, que se adquiere y organiza a través de la propia experiencia y que hace proclive al individuo a reaccionar de una manera característica frente a determinadas personas, fenómenos, objetos o situaciones. Las actitudes han de ser inferidas de las expresiones verbales; y/o de la conducta observada.

Para Sabater (1989), el concepto de actitud es de utilidad en la comprensión de la personalidad, la sociedad, el comportamiento y para orientar la tarea educadora. En su opinión, el concepto ha sido mucho más trabajado desde la perspectiva empírica que la teórica.

Desde una visión general, la actitud se entiende como una predisposición en los sujetos, que se adquiere por aprendizaje y que influye en el comportamiento. La actitud está integrada por elementos conductuales (conativo); de valoración de los estímulos recibidos frente a un determinado comportamiento (afectivo); y de creencias o interpretación de otros estímulos colaterales o sucesivos (cognitivo). Algunas ideas relacionadas plantean que las actitudes son susceptibles de medición. También son modificables pues su adquisición es el resultado de un aprendizaje donde la práctica y la conducta representan los mecanismos fundamentales.

Ante una situación o fenómeno, los sujetos pueden tener varias modalidades de reacción, que se manifiestan de diversas formas pero que pueden analizarse como una "potencia unitaria de reacción". "La actitud vendría a ser un constructo metafísico, un expediente para explicar la coherencia de esas distintas reacciones (conativa, afectiva y cognitiva)" (Sabater, 1989:163). En otras palabras, desde esta perspectiva, la actitud es un promedio de las diversas modalidades de reacción ante un fenómeno; que se inclinaría hacia alguna de ellas de manera preponderante.

Si bien las respuestas de los individuos pueden variar ante el mismo tema, experimentado en una situación real o solamente expresado en una encuesta, se pueden encontrar paralelismos que sirven como indicadores de sus conductas reales manifestadas por las afirmaciones de una escala para medir la actitud frente a tal situación. Es importante tomar en consideración que las respuestas son expresiones verbales ante estímulos que no contienen los esfuerzos, presiones y complejidades que pueden estar presentes en las situaciones reales (Sabater, 1989); sin embargo, no se espera un calco de lo imaginado y la realidad sino información que ofrezca pistas acerca de los comportamientos probables.

Bajo el acuerdo de que los tres componentes antes mencionados (conativo, afectivo y cognitivo) son los conformadores de la actitud, hay posturas que intentan posicionar a alguno o algunos de ellos como su esencia o componente principal. En ese sentido hay dos posturas, la unidimensional, que a su vez contiene a quienes se inclinan por el componente cognitivo (Rokeach, s/f) y otros autores cuya idea es que la orientación afectiva hacia los objetos o situaciones es lo que constituye la base de la actitud, es decir, que la actitud se explica a partir 
de la valoración positiva o negativa que hace el sujeto con respecto al fenómeno (Fishben y Ajzen, 1973).

Por otro lado, están quienes consideran a los tres componentes (conativo, afectivo y cognitivo) como constitutivos de la actitud (Katz y Stotland, 1959); y aquellos otros que desechan al conativo, comprendiendo a la actitud compuesta de manera bidimensional, por lo afectivo y lo cognitivo (Bagozzi y Brunkrant, 1979).

Por su parte, Shove (2003), expone la importancia de los hábitos y rutinas en las prácticas o comportamientos que tiene implicaciones ambientales, y aunque en su texto, se refiere de manera especial al uso del agua y la energía, su modelo contiene elementos que pueden asociarse a otro tipo de actitudes o comportamientos ambientales. Los componentes a partir de los cuales explica las prácticas en el uso de la energía y el agua, los define como las $3 \mathrm{C}$ (Comfort, Cleanliness, Convenience: comodidad, limpieza y conveniencia). A pesar que nuestra atención se centrará en los componentes de la actitud, explicados en los párrafos anteriores, encontramos conveniente expresar que el modelo de Shove, podría adecuarse para explicar las inclinaciones a actuar de una u otra manera en temas ambientales.

En el estudio previo, realizado en el municipio de Teocelo, se manifiesta la importancia de los 3 componentes de la actitud, sin determinar cuál de ellos prevalece sobre los demás. La conducta (conativo) como hábito, es decir la práctica en sí misma de la separación; lo afectivo, manifestado por la percepción positiva o negativa sobre el fenómeno, acompañado al mismo tiempo por sensaciones de aceptación o rechazo del resto del grupo con respecto al acto de separar o de no hacerlo; y la información que poseen los ciudadanos en torno a los beneficios de la separación (cognitivo), influyen en el acto de separar los residuos, y en su intención de continuar haciéndolo (Wojtarowski et al. 2018).

Tomando como base lo anterior, la construcción de la escala de Likert para examinar la actitud de los ciudadanos de Coatepec con respecto a la separación, incluyó afirmaciones relativas a los tres componentes antes mencionados. Se indagó en sus acciones e intenciones con respecto a la separación (elemento conativo), en su información sobre la relación de la separación con el cuidado ambiental y de la salud (cognitivo); así como en la valoración del acto de separación a nivel individual y colectivo (afectivo), para así estimar una potencia unitaria de actitud.

\section{METODOLOGÍA}

\subsection{Técnica: la escala de Likert}

La escala de Likert es un instrumento de recolección de datos para medir actitudes. Se elabora a partir de ítems o frases ante los cuales se solicita la reacción, favorable o desfavorable de los individuos. La medición que se realiza es indirecta, a través de tales ítems, sobre los que los informantes expresan su opi- 
nión (Malave, 2007; Fernández, 1982). En su construcción se les asignan valores a las opciones de respuesta de cada ítem. Los ítems desempeñan el papel de las preguntas o afirmaciones del instrumento. En otras palabras, la escala de Likert es una escala ordinal y nos informa que quien obtiene un puntaje mayor tiene una actitud más favorable que el que tiene un puntaje menor (Malave, 2007).

En nuestro caso la variable es la actitud con respecto a la separación de residuos. El ítem puede plantearse de manera afirmativa o negativa y se refiere al fenómeno o variable que queremos medir. De manera que nuestros ítems se refieren a la separación de residuos. Puntualizamos que, todos los ítems fueron planteados de manera afirmativa.

Con respecto a la definición de los conceptos contenidos en nuestra variable, cuando hablamos de separación, nos referimos a la separación primaria (DOF, 2003), la más básica, que consiste en desechar por un lado lo orgánico y, por otro, lo inorgánico. Entendemos como residuo a cualquier material orgánico o inorgánico generado en los procesos de extracción, beneficio, transformación, producción, consumo, utilización o tratamiento, cuya calidad no permite incluirlo nuevamente en el proceso que lo generó. Los residuos orgánicos son aquellos de origen animal y vegetal: cáscaras de frutas y verduras, cascarones de huevo, sobrantes de comida, servilletas de papel usadas, residuos de café, bolsitas de té, pasto, hojas, ramas y flores, entre otros. Los residuos inorgánicos son bolsas, empaques y envases plásticos, vidrio, papel, cartón y metales; aquí también se incluyen los sanitarios (papel higiénico, pañuelos faciales, algodón, pañales, toallas sanitarias y condones) y especiales (pilas, focos, electrónicos, radios, teléfonos, televisores, teclados...) y envases de pegamento, aceite de motor, aerosoles, cosméticos, tintes y fijadores para el cabello, pintura, entre otros. (SEMARNAT, 2009).

\subsection{El instrumento}

Se diseñó una Escala de Likert como instrumento, que fue aplicada en prueba piloto a 7 residentes en el municipio de Coatepec. El instrumento contó con 11 afirmaciones y las mismas 5 opciones de respuesta (Totalmente de acuerdo, De acuerdo, Ni de acuerdo ni en desacuerdo, En desacuerdo, Totalmente en desacuerdo) para cada una. Se solicitó a los informantes que la respondieran y además que expresaran su opinión sobre la comprensión de las afirmaciones. A partir de los resultados de esta prueba se definió que la escala definitiva quedaría en 10 ítems, omitiendo una frase que estaba planteada en modo negativo y resultaba problemática su comprensión. Se incluyeron elementos de mejora en el resto de las afirmaciones.

Las afirmaciones asociadas a la variable que se midió y a los componentes que estamos considerando de la actitud, se muestran en el cuadro 1. De las 9 afirmaciones, 3 están asociadas al componente conativo, 3 al cognitivo y otras 3 al afectivo. Como pudo verse: 
Los ítems 3, 4 y 7 se asocian al componente conativo;

Los ítems 1, 2 y 5 al componente cognitivo y, finalmente, los ítems 6,8 y 9 y 10 al componente afectivo.

Cuadro 1: Afirmaciones asociadas a variable y componentes

\begin{tabular}{|l|c|}
\hline \multicolumn{2}{|c|}{ VARIABLE (SEPARACIÓN DE RESIDUOS) } \\
\hline \multicolumn{1}{|c|}{ AFIRMACIONES O ÍTEMS } & COMPONENTE \\
\hline $\begin{array}{l}\text { 1.La separación de los residuos ayuda a prevenir la } \\
\text { contaminación }\end{array}$ & Cognitivo \\
\hline $\begin{array}{l}\text { 2. La separación de los residuos ayuda a prevenir problemas de } \\
\text { salud entre la población }\end{array}$ & Cognitivo \\
\hline 3. Yo ya separo los residuos que genero & Conativo \\
\hline 4. Me gustaría separar los residuos & Conativo \\
\hline $\begin{array}{l}\text { 5. Sé diferenciar los residuos orgánicos de los inorgánicos } \\
\text { 6. Me gustaría vivir en un municipio donde se separen los } \\
\text { residuos }\end{array}$ & Afectivo \\
\hline $\begin{array}{l}\text { 7. En mi municipio se debería recolectar por separado lo } \\
\text { orgánico de lo inorgánico }\end{array}$ & Conativo \\
\hline $\begin{array}{l}\text { 8. Mi municipio debería tener un programa de composta y hacer } \\
\text { abono con los residuos orgánicos. }\end{array}$ & Afectivo \\
\hline $\begin{array}{l}\text { 9.El uso de abono orgánico puede beneficiar a los productores } \\
\text { de café, caña y otros cultivos de mi municipio }\end{array}$ & Afectivo \\
\hline
\end{tabular}

Fuente: Elaboración propia (2018).

En conjunto nos darán una respuesta unitaria o general sobre la actitud de nuestros informantes con respecto a la variable estudiada. Entre todos conforman nuestra escala para medir la actitud con respecto a la separación de residuos. Estas 9 afirmaciones conformaron la escala para medir la actitud hacia el fenómeno separación de residuos. Contamos además con otra afirmación que se presenta por separado y se refiere a la opinión sobre el funcionamiento del sistema de limpia pública municipal. Esta última afirmación a pesar de estar vinculada al tema de estudio, no correspondía a la categoría a la cual quería darse respuesta. Sus resultados se muestran por ser útiles para una mejor comprensión del fenómeno en Coatepec. Así, la opinión sobre la gestión de residuos por parte del municipio de Coatepec, se presenta a través de una única afirmación (Cuadro 2). 


\section{Cuadro 2: Afirmación sobre el funcionamiento del sistema de limpia pública municipal}

\begin{tabular}{|l|l|}
\hline \multicolumn{1}{|c|}{ AFIRMACIÓN } & \multicolumn{1}{c|}{ VARIABLE } \\
\hline $\begin{array}{l}\text { Mi municipio atiende bien el tema de la } \\
\text { basura }\end{array}$ & $\begin{array}{l}\text { Opinión con respecto al funcionamiento } \\
\text { del sistema de limpia pública municipal }\end{array}$ \\
\hline
\end{tabular}

Fuente: Elaboración propia (2018)

Todos los ítems correspondieron a afirmaciones planteadas de manera positiva, en cuanto a los puntajes de cada una de las respuestas, se dio de la siguiente forma:

Cuadro 3: Puntajes según respuestas a las afirmaciones

\begin{tabular}{|c|c|c|c|c|}
\hline $\begin{array}{c}\text { Totalmente } \\
\text { de acuerdo }\end{array}$ & De acuerdo & $\begin{array}{c}\text { Ni de } \\
\text { acuerdo ni en } \\
\text { desacuerdo }\end{array}$ & En desacuerdo & $\begin{array}{c}\text { Totalmente } \\
\text { en } \\
\text { desacuerdo }\end{array}$ \\
\hline 5 & 4 & 3 & 2 & 1 \\
\hline
\end{tabular}

Fuente: Elaboración propia (2018)

Los puntajes son los valores asignados a las opciones de respuesta. El número mayor representa una reacción más favorable con respecto a la afirmación y a la categoría que se intenta medir. Conforme el número es menor la actitud es más desfavorable u opuesta. Para obtener el Puntaje Total (PT) de cada informante se suman los valores que obtuvo respecto de cada ítem. El puntaje mínimo resulta de la multiplicación del número de ítems por 1, que es el valor más bajo que puede asignarse a cada afirmación. El puntaje máximo está dado por el número de ítems o afirmaciones multiplicado por 5, que es el valor más alto entre las respuestas.

De manera que para la variable separación de residuos, que consta de 9 afirmaciones, los informantes podrían obtener un puntaje total (PT) máximo de 45 y uno mínimo de 9 . Al medir por separado los ítems correspondientes a cada uno de los componentes de la actitud, en todos los casos, el PT máximo corresponde a 15 y el mínimo a 3, pues cada una de estas secciones cuenta con 3 afirmaciones.

Por su parte, en la medición de la "opinión con respecto al funcionamiento del sistema de limpia pública municipal", el PT máximo corresponde a 5 y el mínimo a 1, porque se trata solamente de una afirmación. Es necesario aclarar que, en el caso de esta frase solitaria, a pesar de que fue presentada en la escala general, se está considerando que la respuesta que daremos corresponde a una única idea, no a un conjunto de ítems para inferir la respuesta a una variable. Nos 
permitimos esta licencia, por considerar que la respuesta a esta única afirmación puede aportar información confiable y de interés en el tema abordado, de manera que se incluyó en el instrumento, aunque sus resultados se presenten por separado de la medición de la actitud ante la separación de residuos.

Los resultados se separaron en quintiles $(\mathrm{Q})$, definidos a partir del puntaje total de cada informante. El Q 1 representa la actitud más desfavorable hacia la categoría estudiada y el 5 la más positiva. Para la actitud en general (considerando los 3 componentes), que contiene los 9 ítems, los quintiles quedaron como se muestra en el siguiente cuadro (4).

Cuadro 4: Rangos entre quintiles para la medición general de la actitud

\begin{tabular}{|c|c|}
\hline Quintil & Puntaje Total \\
\hline 1 & $9-16.2$ \\
\hline 2 & $16.3-23.4$ \\
\hline 3 & $23.5-30.6$ \\
\hline 4 & $30.7-37.8$ \\
\hline 5 & $37.9-45$ \\
\hline
\end{tabular}

Fuente: Elaboración propia (2018).

En el caso de los componentes por separado, cada uno cuenta con 3 ítems, de manera que los rangos entre quintiles son iguales para todos, como puede observarse en el cuadro 5.

La escala se aplicó a 108 individuos (68 mujeres y 40 hombres) y los criterios de aplicación fueron que contaran con la mayoría de edad y ser residentes del municipio de Coatepec. Se trata de una muestra no probabilística, donde se fueron analizando las encuestas en cuanto iban siendo respondidas. Así, el fenómeno de saturación se dio rápidamente, sin embargo, se siguió avanzando a la espera de encontrar variabilidad en las respuestas, pero al continuar lo homogeneidad decidió detenerse en la encuesta 108.

Este tipo de muestra se considera adecuada para un estudio exploratorio como el que aquí se presenta.

Cuadro 5: Rangos entre quintiles para la medición por separado de cada componente

\begin{tabular}{|c|c|}
\hline Quintil & Puntaje Total \\
\hline 1 & $3-5.4$ \\
\hline 2 & $5.5-7.8$ \\
\hline 3 & $7.9-10.2$ \\
\hline 4 & $10.3-12.6$ \\
\hline 5 & $12.7-15$ \\
\hline
\end{tabular}

Fuente: Elaboración propia (2018). 


\subsection{Método de confiabilidad de la Escala de Likert}

La confiabilidad se refiere al grado en que un instrumento realmente mide la variable que pretende medir (Hernández et al. 2010). En nuestro caso, para la estimación de la confiabilidad de la escala tipo Likert, se aplicó la medida de consistencia interna alfa de Cronbach (1951). El cálculo mostró un coeficiente de 0.704, para 9 ítems, puntaje aceptable en estudios de validación de instrumentos de encuesta (Polit y Hungler, 2000).

\subsection{Análisis estadístico}

Los análisis estadísticos fueron realizados con SPSS (Satatistical Package for the Social Sciences) versión 19 para windows. Se realizó el análisis de la normalidad de los datos con la prueba de Kolmogorov-Smirnov. Los datos no fueron normales por lo que se utilizaron pruebas no paramétricas. Se empleó el análisis de Kruskal- Wallis para detectar diferencias estadísticas entre las respuestas, cuando estas fueron confirmadas se compararon los efectos significativos mediante la prueba de de Mann-Whitney con una probabilidad de 0.01 .

\subsection{Características de la muestra}

Como vimos antes, la encuesta fue respondida por 68 mujeres y 40 hombres, es decir 63\% de la muestra corresponde a las primeras y $37 \%$ a los últimos.

En cuanto a otras características de los informantes, a continuación, puede verse la correspondencia de los individuos con su escolaridad (Figura 1).

La mayoría de los informantes cuentan con una licenciatura como último grado de estudios, seguidos por aquellos que se encuentran cursando la carrera y en tercer lugar están quienes tienen una maestría. En menor cantidad encontramos el resto de los grados de estudio. Todo esto indica que es una muestra con una fuerte presencia de individuos que han contado con largos años de educación formal. 


\section{Grado de estudios}

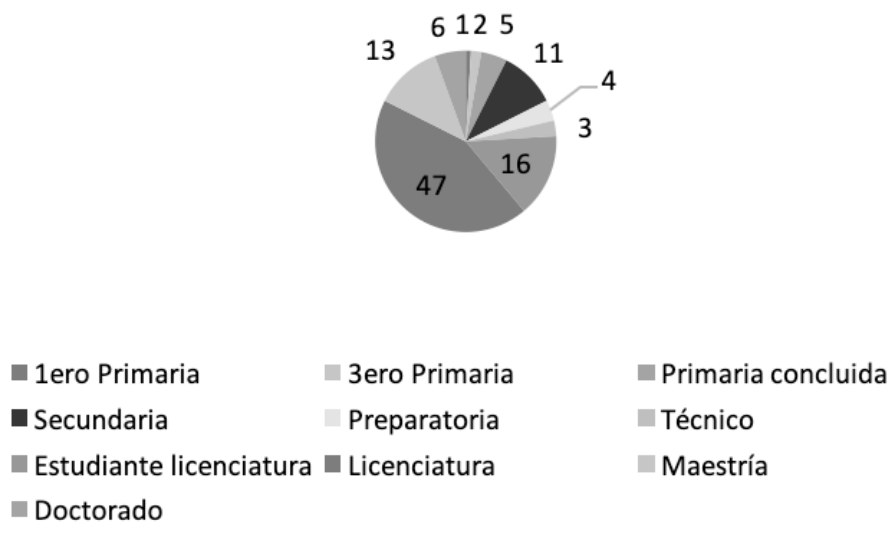

Figura 2. Grado de estudios

Fuente: Elaboración propia (2018)

Los participantes habitan en diversas colonias del municipio; con base en la ubicación geográfica de las mismas, entre todos, representan al Centro de la cabecera municipal, y otras 28 zonas (Cuadro 6).

Cuadro 6. Zonas donde habitan los informantes

\begin{tabular}{|l|l|l|}
\hline Zona & $\begin{array}{l}\text { Ubicación geográfica con } \\
\text { respecto al centro de la } \\
\text { cabecera municipal }\end{array}$ & $\begin{array}{l}\text { Re s iden te s } \\
\text { encuestados }\end{array}$ \\
\hline Centro & Centro & 42 \\
\hline Campo Viejo & Sureste & 12 \\
\hline Emiliano Zapata & Suroeste & 11 \\
\hline La Gachupina & Norte & 5 \\
\hline La Pitaya & Noreste & 4 \\
\hline 22 de septiembre & Este & 4 \\
\hline Plan de Minas & Norte & 4 \\
\hline Francisco Villa & Noreste & 3 \\
\hline Bernardo Casals & Sur & 2 \\
\hline Quiebrahacha & Oeste & 2 \\
\hline Las Lomas & Noroeste & 1 \\
\hline
\end{tabular}




\begin{tabular}{|l|l|l|}
\hline Zona & $\begin{array}{l}\text { Ubicación geográfica con } \\
\text { respecto al centro de la } \\
\text { cabecera municipal }\end{array}$ & $\begin{array}{l}\text { Re s i d e n t e s } \\
\text { encuestados }\end{array}$ \\
\hline Mahuixtlán & Sureste & 1 \\
\hline San Pedro & Noreste & 1 \\
\hline Espinal alto & Oeste & 1 \\
\hline La Orduña & Este & 1 \\
\hline 2 de abril & Oeste & 1 \\
\hline Las Hayas & Noroeste & 1 \\
\hline Lázaro Cárdenas & Noroeste & 1 \\
\hline Infonavit & Sur & 1 \\
\hline Cerro Súchil & Suroeste & 1 \\
\hline Espinal Bajo & Oeste & 1 \\
\hline I. Allende & Noreste & 1 \\
\hline Arenales & Suroeste & 1 \\
\hline El Porvenir & Noreste & 1 \\
\hline Tricentenario & Sur & 1 \\
\hline Pacho Viejo & Noreste & 1 \\
\hline Plan de Mavil & Oeste & 1 \\
\hline 16 de septiembre & Sur & 1 \\
\hline Juan de la Luz Enríquez & Sur & 1 \\
\hline
\end{tabular}

Fuente: Elaboración propia (2018)

Como puede verse la mayoría de los encuestados residen en la Colonia Centro de la cabecera municipal de Coatepec y el resto se distribuye por todos los puntos cardinales alrededor del centro de la ciudad. En este sentido, reconocemos que ciertas características de la muestra, como el alto nivel educativo, están influidas por el número de informantes residentes en la colonia Centro, dado que en general, las periferias de las ciudades mexicanas concentran a los ciudadanos más desfavorecidos económicamente y con menos acceso a niveles educativos superiores. De manera que nuestros resultados, aunque aportan una idea general sobre la ciudad, representan sobre todo, la opinión de los residentes del centro.

\subsection{Análisis por componente}

En el análisis por separado de cada uno de los 3 componentes de la actitud, puede observarse de manera general, regularidad en las respuestas, que se ubican 
en su mayoría entre la opinión "muy favorable" y "favorable"; es decir en los quintiles 5 y 4 , respectivamente.

\subsubsection{Componente conativo}

A continuación, puede verse el análisis específico del componente conativo, éste se encuentra asociado a las acciones y/o intenciones de separación de residuos por parte de los informantes, se observa que poco menos de $60 \%$ de ellos se ubica en el quintil 5 , algo más de $35 \%$ en el 4 y un porcentaje mucho menor en el 3 (figura 2), los cuales fueron estadísticamente diferentes entre ellos ( $\mathrm{p}=0$. 001).

\section{Componente conativo}

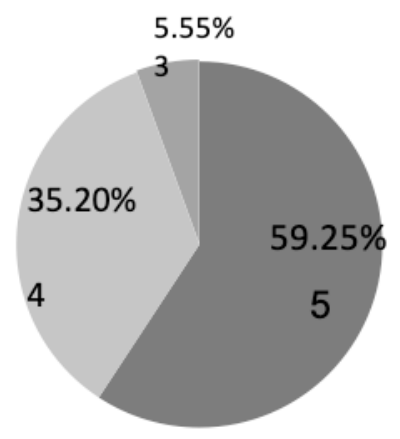

Figura 3. Componente conativo

Fuente: Elaboración propia (2018).

A partir de esto puede decirse que, con respecto a la toma de acciones directas o intenciones de separar los residuos, la mayoría de los informantes se encuentran en una posición muy favorable, seguidos por los que tienen una postura favorable y en menor medida se presentan algunos con una postura intermedia o indiferente. Lo anterior indica que existe, en general, una alta disposición para la separación. Para corroborarlo sería pertinente que en estudios futuros y de largo plazo se analice si tal intención de separar los residuos, se ha realizado. Chuliá (1995) propuso que los comportamientos individuales y colectivos también deben ser considerados (dimensión activa) al momento de medir valores de conciencia ambiental. Para el caso específico de este estudio, esta dimensión puede ser considerada en un estudio de seguimiento.

Para el caso del casi 6\% de individuos que permanecen indiferentes a la intención de separar la basura, sería viable seguir promoviendo acciones para la concientización sobre el buen manejo de los residuos sólidos, donde se considere 
la aplicación del conocimiento en situaciones reales de vida, tal y como señalan Dieleman y Juárez Nájera (2008) quienes promueven la educación y concientización ambiental encaminada a la sustentabilidad basadas en la interacción del quehacer científico y del conocimiento empírico y participación de la gente. Este componente estuvo constituido por 3 afirmaciones, el análisis de todas ellas, en conjunto, nos proporcionó los resultados antes expuestos, sin embargo, nos resulta pertinente exponer el resultado específico de una de estas 3 afirmaciones porque muestra las respuestas sobre el hecho concreto de separar los residuos. Este dato aporta información valiosa sobre quienes ya tienen este hábito.

En la siguiente figura (4) se muestran las respuestas a la afirmación "Yo ya separo los residuos que genero". Para tal caso, se observaron diferencias significativas entre los distintos quintiles $(\mathrm{p}=0.006)$.

Quienes separan y quienes no separan sus residuos

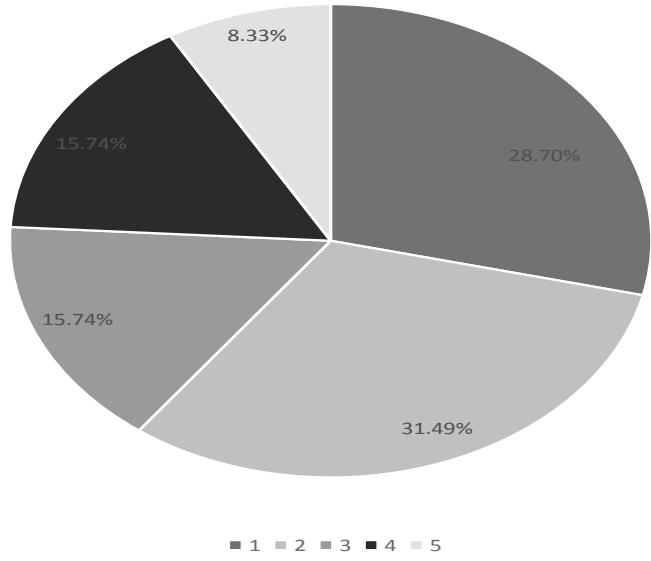

Figura 4. Quienes separan y quienes no separan sus residuos

Fuente: Elaboración propia (2018)

También en este caso, los quintiles 5 y 4 muestran el mayor grado de acuerdo con la afirmación, entre los cuales no hubo diferencias estadísticas ( $\mathrm{p}=$ 0.001). Puede verse que la mayoría de las respuestas se ubican en el Q4, seguidas por el Q5.

Lo anterior significa que, en total, poco más de $60 \%$ de los informantes expresa que ya realizan acciones de separación de residuos, $28.70 \%$ de ellos lo manifiesta con más seguridad ubicándose en la postura correspondiente al Q más alto, y $31.49 \%$ asume que ya lo hace pero no se atreve a dar la respuesta más favorable, quizás porque tienen dudas con respecto a si es adecuada o no su manera de realizarlo o porque consideran que realizan una separación muy básica. 
Por su parte los Q 1, 2 y 3 presentan valores menores a los Q 4 y 5 ( $\mathrm{p}<0.01)$, sin embargo no difieren estadísticamente entre ellos ( $p>0.01$ ), $8.33 \%$ reconoce que no separa nada en absoluto, y $15.74 \%$ considera que no lleva a cabo acciones de separación, otro $17.74 \%$ se mantiene al margen de responder un grado favorable o desfavorable de acuerdo y caen en la categoría que podríamos llamar "indiferente".

Los datos indican que más de la mitad de los individuos encuestados reconoce que lleva a cabo acciones de separación de residuos. Estimamos que este es un porcentaje considerable si se toma en cuenta que no existe la contraparte gubernamental para gestionar los residuos de manera separada. No indagamos en las causas de tal acto, pero podemos adelantar que existen conocimientos de los beneficios sociales y ambientales de la separación, así como conciencia de la importancia de tomar acciones proambientales.

\subsubsection{Componente cognitivo}

A continuación, presentaremos los resultados concernientes al componente cognitivo, que está asociado a la información con respecto a la relación entre separación de residuos y cuidado del ambiente y de la salud, así como el conocimiento sobre el carácter de los residuos, que permitiría una separación básica adecuada.

En la figura 5 puede observarse que, con respecto al componente cognitivo, existe un porcentaje considerable de informantes (poco más de 86\%; Q5) que se encuentra en el nivel más alto de acuerdo con las afirmaciones relativas a su conocimiento sobre los beneficios de la separación y sobre la identificación del tipo de residuos, dicho quintil es estadísticamente mayor $(\mathrm{p}<0.01)$ a los quintiles 3 y 4, estos últimos a su vez también difieren estadísticamente entre sí $(\mathrm{p}=0.020)$.

\section{Componente cognitivo}

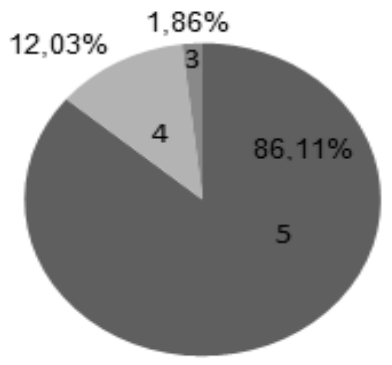

Figura 5. Componente cognitivo

Fuente: Elaboración propia (2018). 
Se infiere que hay un número considerable de informantes que tienen conocimientos básicos sobre el tema, en su relación con los perjuicios ambientales y de la salud humana.

\subsubsection{Componente afectivo}

En cuanto al componente afectivo, el análisis de los datos muestra que un elevado porcentaje de la muestra se ubica en el quintil más alto de acuerdo con las afirmaciones de este componente, quedando en un muy rezagado segundo y tercer lugar los quintiles 4 y 3 (figura 6). Este componente ha resultado el que tiene un grado mayor de posturas muy favorables. Sus afirmaciones se asocian al deseo de vivir en un municipio donde se lleve a cabo la separación de residuos y donde se elabore composta con los residuos orgánicos, comprendiendo que esta composta puede ser benéfica para los productores agroforestales (café) del municipio.

\section{Componente afectivo}

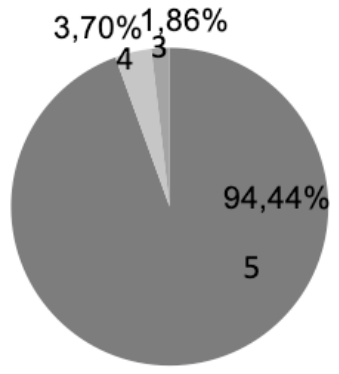

Figura 6. Componente afectivo

Fuente: Elaboración propia (2018).

De manera que, además de la satisfacción personal de vivir en un municipio que gestione adecuadamente sus residuos, se pone de manifiesto un sentido de comunidad y empatía con la gente de la región, específicamente con productores que pueden hacer uso del abono que se pueda generar en una planta de lombricompostaje. 


\subsection{Actitud ante la separación de residuos}

El análisis de los datos en conjunto (9 ítems) muestra que, para la variable de separación de residuos, los encuestados se dividen entre el quintil (Q) 5 y el Q 4, la mayoría de ellos en el Q5. A continuación se puede observar (figura 7) el porcentaje de informantes ubicado en cada quintil.

\section{Actitud ante la separación}

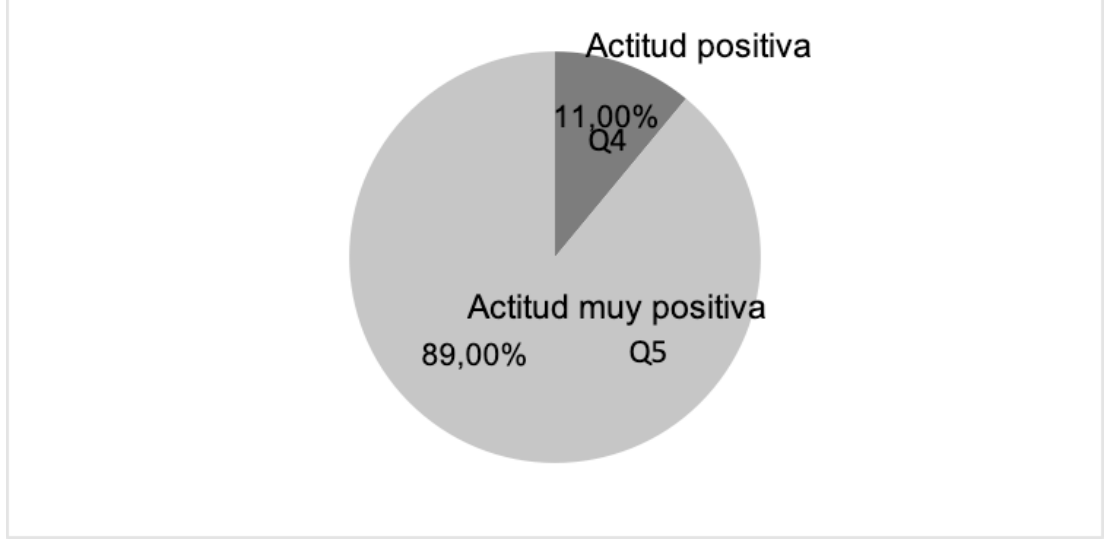

Figura 7: Actitud ante la separación

Fuente: Elaboración propia (2018).

Como puede verse, un alto porcentaje (89\%) de la muestra manifiesta una actitud muy positiva ante la separación de residuos, pues se ubican en el Q más alto posible $(\mathrm{p}=0.001)$, mientras que el resto $(11 \%)$ presentan una actitud positiva frente a la misma variable.

Es importante destacar que la ubicación en los quintiles más altos (Q 5 y 4) refleja la actitud más favorable y favorable, respectivamente, con respecto a la separación. De todos los PT, el más bajo fue de 32, que como puede encontrarse en la sección de Metodología, corresponde al Q4; lo que significa que no encontramos informantes ubicados en los quintiles 3,2 y 1 ; los cuales representan, respectivamente, una actitud: indiferente, desfavorable y muy desfavorable.

Lo anterior nos indica que parece haber una disposición positiva por parte de estos individuos para separar los residuos, de manera que sería relativamente fácil que llevaran a cabo esta acción (los que aún no la realizan), porque como se mostró antes, un porcentaje importante de los encuestados (poco más de 60\%) ya separa los residuos que genera. 
Arriba mencionamos que este porcentaje nos parecía significativo tomando en cuenta que no hay un programa municipal de gestión de RSU.

En ese sentido, a continuación, expondremos el resultado que se obtuvo para la afirmación relativa a la opinión de los encuestados con respecto a cómo se atiende el tema de la basura en el municipio de Coatepec.

\subsubsection{Opinión sobre la atención del tema de la basura en el municipio de Coatepec}

Desde una perspectiva general, hay una opinión desfavorable sobre la gestión de residuos que realiza el municipio de Coatepec. En la siguiente figura (8) podemos observar la distribución por quintiles según las respuestas de nuestros informantes.

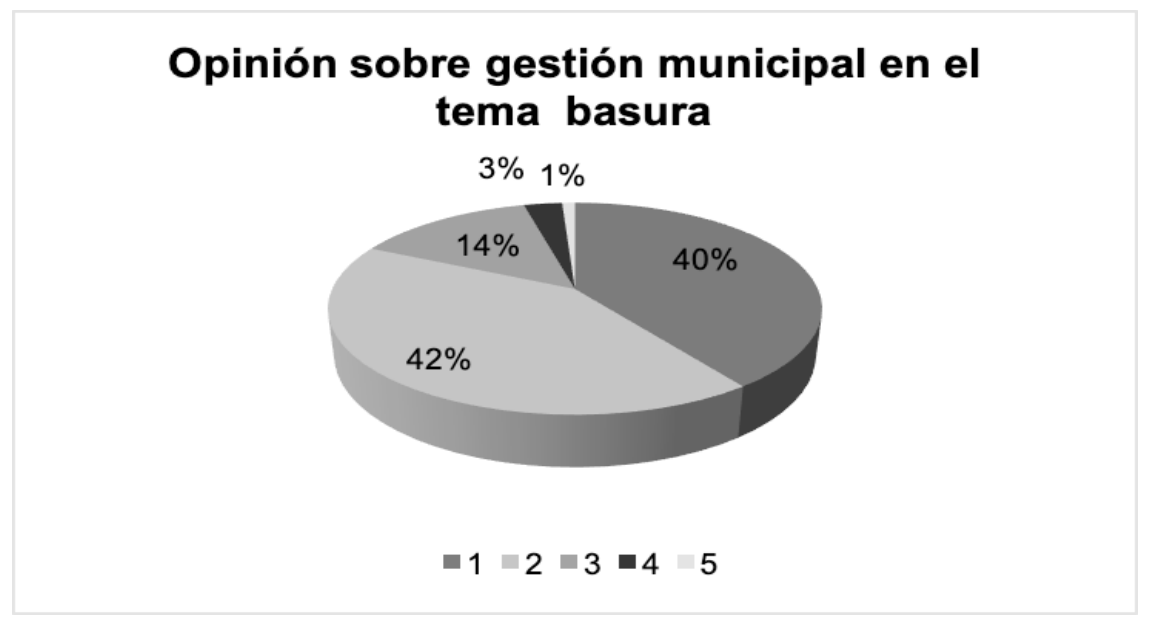

Figura 8. Opinión sobre gestión municipal en el tema de la basura

Fuente: Elaboración propia (2018).

Se aprecia que el $40 \%$ de la muestra se ubica en el Q1, con una opinión muy desfavorable y el $42 \%$ en el 2, con una actitud desfavorable; lo que significa que el $80 \%$ considera que es muy mala o mala la manera en que se atiende el tema de la basura en el municipio.

Los Q3, 4 y 5 muestran porcentajes mucho menores; $14 \%$, 3\% y $1 \%$, respectivamente; en el orden planteado, tales Q representan quienes tienen una opinión que calificamos como indiferente, favorable y muy favorable. De manera que solamente $4 \%$ de la muestra manifiesta que el municipio atiende bien o muy bien 
el tema de la basura. Esta opinión puede representar una llamada de atención para buscar alternativas de mejora en este sentido.

\subsection{Análisis de resultados desde el concepto de Actitud}

En los resultados se aprecia congruencia entre los componentes conativo, cognitivo y afectivo como conformadores de la actitud: observamos que se comportan de una manera similar, en el sentido de que todos arrojan niveles altos o muy altos de acuerdo por parte de los informantes. A partir de esto, estimamos que, en nuestra investigación, la postura que explica el fenómeno de la actitud es la de Katz y Stotland (1959), quienes tienen esta visión tripartita del contenido de la actitud. Sin embargo, no hay que desestimar otras ideas que también están representadas en nuestros resultados.

A continuación, exponemos un cuadro (7) que muestra los porcentajes para cada $\mathrm{Q}$ por componente, y enseguida ampliamos nuestro análisis.

\section{Cuadro 7. Porcentajes para cada $Q$ por componente}

\begin{tabular}{|c|c|c|c|}
\hline & \multicolumn{3}{|c|}{ Componente } \\
\hline Quintil & Conativo & Cognitivo & Afectivo \\
\hline 5 & $59.25 \%$ & $86.11 \%$ & $94.44 \%$ \\
\hline 4 & $35.20 \%$ & $12.03 \%$ & $3.70 \%$ \\
\hline 3 & $5.55 \%$ & $1.86 \%$ & $1.86 \%$ \\
\hline
\end{tabular}

Fuente: Elaboración propia (2018).

Planteamos que la visión tripartita es de utilidad en nuestro análisis porque se observa que los tres componentes muestran porcentajes importantes en los niveles más altos de acuerdo con respecto a cada componente y por consecuencia, a nuestra categoría general de separación de residuos. No obstante, es importante destacar que sin que nuestro afán sea determinar cuál de ellos prevalece sobre los otros, los datos exponen que el componente afectivo presenta un porcentaje significativamente más alto en el Q correspondiente a "muy favorable" que el resto, coincidiendo con la postura de Fishben y Ajzen (1973), quienes consideran que este componente constituye la base de la actitud.

Al mismo tiempo, los resultados arrojan, en cierta medida, cercanía a la visión bidimensional de Bagozzi y Brunkrant, (1979), donde se asume que son los componentes cognitivo y afectivo los que explican mejor la actitud, y es que podemos ver que a pesar de que el afectivo arroja un porcentaje mayor ( $94.44 \%)$ en el máximo nivel de acuerdo, también el cognitivo cuenta con cifras muy altas 
(86.11\%); ambos con resultados para ese Q, significativamente más altos que el componente conativo, que tiene $59.25 \%$.

Para matizar nuestra mención a la postura bidimensional, aclaramos que ésta no toma en cuenta el componente conativo, y nuestro estudio refleja que, si bien éste no alcanza las cifras de los otros dos, su porcentaje es considerable.

En nuestro caso, consideramos que la visión tripartita ha sido adecuada para explicar la actitud, no ignoramos la posibilidad de que algunos componentes puedan tener mayor peso que otros, pero nos resulta pertinente la presencia de todos; es decir, afirmamos que en esta explicación no debe ser excluido el componente conativo.

El resultado general de la actitud sobre separación de residuos, resulta de la combinación de resultados de los 3 componentes que hemos analizado. Como vimos antes, para la actitud general una gran mayoría de los informantes se ubicó en el Q5 (89\%), seguidos del Q4 (11\%), lo cual representa una actitud muy favorable y favorable, respectivamente, en referencia a la separación. Y pone de manifiesto que cada uno de los componentes influyó en el resultado general. Por lo que hay congruencia entre los datos arrojados sobre cada uno de ellos con el resultado final.

A pesar de que en este caso el objeto de la actitud fue la separación de residuos, podríamos ir más allá y expresar que la ciudadanía encuestada en Coatepec está dispuesta a un cambio social a partir de la transformación de sus hábitos y prácticas en el terreno del manejo de residuos. Que al mismo tiempo anhela también un cambio en las políticas públicas municipales en el mismo tema, pero cuyas consecuencias van más allá de la pura gestión de los residuos y que se trata de mejorar las condiciones socioambientales de su localidad. Parecen dadas las condiciones para que estos acuerdos entre ciudadanía y gobierno municipal, pueden construirse a partir de un proceso de gobernanza, contextualizado, con acciones colectivas conscientes para transitar hacia un estado deseable (García, 2006).

En las respuestas del componente afectivo, los ciudadanos ponen de manifiesto este deseo de transformación, así como una actitud que traspasa los beneficios individuales y que puede situarse en la idea de valores colectivos y de solidaridad con otros grupos, como son los productores de su municipio.

De esta manera, es importante destacar la factibilidad de este método para examinar las actitudes pro-ambientales desde una perspectiva más amplia.

Por otro lado, vinculando los resultados con las ideas de Shave (2003), puede decirse que el componente afectivo, que presenta porcentajes altos en el Q5 "muy favorable", se asocia a su vez a los componentes del modelo de esa autora. La comodidad, la limpieza y la conveniencia se relacionan con sensaciones positivas que han sido construidas a partir de hábitos y prácticas. 


\section{CONCLUSIONES}

Se concluye que los tres componentes de la actitud muestran porcentajes importantes en los quintiles más altos, tanto en Q5 como en Q4, mayormente en el primero de ellos.

En el componente conativo, casi $60 \%$ se encuentra en el Q5, lo que indica que más de la mitad de los informantes ya toma acciones de separación o muestra intenciones de hacerlo.

En el componente cognitivo, poco más de $86 \%$ se encuentra en el nivel más alto de acuerdo (Q5) con las afirmaciones relativas a conocimientos sobre los beneficios de la separación y sobre la identificación del carácter primario de los residuos. Esto muestra un presunto conocimiento de estos informantes sobre el tema de residuos y su primer nivel de separación (orgánico e inorgánico).

En cuanto al componente afectivo, un elevado porcentaje de la muestra (94.44\%) se ubica en el quintil más alto de acuerdo, siendo este componente el que resultó con un grado mayor de posturas muy favorables.

En cuanto a las dos afirmaciones cuyas respuestas se presentaron también por separado, se vio que poco más de $60 \%$ de los informantes expresa que ya realizan acciones de separación de residuos. En cuanto a la opinión sobre la gestión de los residuos por parte de la autoridad municipal, se aprecia que la mayoría de los informantes se ubicaron en los Q más bajos (1y 2), 42\% en el Q2 con una opinión muy desfavorable y $40 \%$ en el Q1, con una opinión desfavorable; lo que significa que más de $80 \%$ considera que es mala o muy mala la manera en que se gestiona el tema de la basura en el municipio.

Con respecto a la actitud general para la separación de residuos, se vio que todos los informantes se ubicaron en los Q más altos, $89 \%$ en el Q5 y $11 \%$ en el Q4; lo que pone de manifiesto una actitud muy favorable y favorable, respectivamente. Con base en todo lo analizado, tomando en cuenta las acciones de separación que ya lleva a cabo una parte de los ciudadanos, la postura favorable de la mayoría para separar y que el municipio gestione bajo esa lógica los residuos, así como la opinión negativa de un porcentaje importante de la muestra con respecto a cómo gestiona Coatepec actualmente el tema de la basura; puede considerarse que existe una disposición positiva de la ciudadanía para participar en un programa municipal de gestión integral de residuos bajo un esquema de gobernanza. Estimamos que se facilitaría al menos la separación primaria, que es el paso inicial o básico para el funcionamiento de un proceso de esta naturaleza.

El análisis realizado con base en las dimensiones conativa, cognitiva y afectiva, nos muestra a su vez un panorama de lo que falta por entender y atender en la población con respecto a la separación y buen manejo de los residuos sólidos desde el nivel individual, colectivo y de los representantes políticos, lo cual resulta una línea base importante para el diseño de estrategias de planeación y de educación ambiental.

Sería pertinente realizar estudios donde se relacione a mayor profundidad los componentes de la actitud que han guiado esta investigación con los componen- 
tes del modelo de Elizabeth Shave, en el análisis de comportamientos y prácticas ambientales en general.

\section{BIBLIOGRAFÍA}

Acurio, G., Rossin, A., Texeira, P. y Zepeda, F. (1997). Diagnóstico de la situación de residuos sólidos municipales en América Latina y el Caribe. Washington: Banco Interamericano de Desarrollo y la Organización Panamericana.

Ajzen, I. (1991). The theory of planned behavior. Organ. Behavior and Human Decision Processes 50 179-211.

Armijo, C., Puma, A., y Ojeda, S. (2012). El conocimiento de los habitantes de una ciudad mexicana sobre el problema de la basura. Revista internacional de contaminación ambiental, 28, 29-37.

Banco Mundial. (2012). What a Waste: A Global Review of Solid Waste Management. Recuperado de: https://siteresources.worldbank.org/INTURBANDEVELOPMENT/ Resources/336387-1334852610766/What_a_Waste2012_Final.pdf

Bagozzi, R.P. y Burnkrant, R.E. (1979). Attitude organization and the arritude-behavior relationshíp. J. Per. Soc. Psychol., 37, pp. 913-929.

Berkes, F.; Colding, J. y C. Folke (ed.) (2003): Navigating Social-Ecological Systems: Building Resilience for Complexity and Change. Cambridge, Cambridge University Press.

Boldero, J. (1995). The prediction of household recycling of newspaper: The role of attitudes, intentions and situational factors. Journal of Applied Social Psychology, 25, 440-462.

Cadena, C. E., Moreno, S., del Pilar, R., y Armenteras Pascual, D. (2007). Evaluación del estado de los bosques de niebla y de la meta 2010 en Colombia. Instituto de Investigación de Recursos Biológicos Alexander von Humboldt.

Chu, P. y Chiu, J. (2003). Factors influencing household waste recy- cling behavior: Test of an integrated model. Journal of Ap-plied Social Phychology, 33, 604-626.

Chuliá, E. (1995). La conciencia medioambiental de los españoles en los Noventa. ASP Research Paper, 12 (a), 1-36.

Cronbach, L. J. (1951). Coefficient alpha and the internal structure of tests. Psychometrika. 16, 297-334.

Daily, G. (1997). Nature's services: Societal dependence on natural ecosystems. Island Press, NuevaYork, EUA. 392 p.

Diario Oficial de la Federación (DOF). (2003). Ley General para la Prevención y Gestión Integral de Residuos. Cámara de Diputados del H. Congreso de la Unión. Recuperado de: file:///F:/Mis\%20documentos/Posdoctorado/FORMALIA/MARCO_NORMATIVO/ley\%20de\%20residuos\%20(2).pdf

Dieleman, H. y M. Juárez-Nájera. 2008. ¿Cómo se puede diseñar educación ambiental para la sustentabilidad? Revista internacional de contaminación ambiental 24(4):131-147.

Durán, M., Alzate, M., y Sabucedo, J.M. (2009). La influencia de la norma personal y la teoría de la conducta planificada en la separación de residuos. Medio Ambiente y Comportamiento Humano, 10 (1y2), 27-39.

Escobar, A. (1994): Encountering Development: The Making and Unmaking of the Third World. Ewing (NJ), Princeton University Press. 
Fernández, I. 1982. Construcción de una escala tipo Likert. Ministerio de Trabajo y Asuntos Sociales, España e Instituto Nacional de seguridad e higiene en el trabajo. Recuperado de: http://www.insht.es/InshtWeb/Contenidos/Documentacion/FichasTecnicas/NTP/Ficheros/001a100/ntp_015.pdf

Fishben, M. y Ajzen, 1. (1973). Attitudes toward objects as predictors of single and multiple behavioral criteria. Psychol. Rev. 23 (1974); pp. 59-74.

García, E. (2006). "El cambio social más allá de los límites al crecimiento: un nuevo referente para el realismo en la sociología ecológica", en Espinoza, L. E.uis y Cabero Valentín (ed.): Sociedad y medio ambiente, 53-74, Universidad de Salamanca, Salamanca.

Hernández, R., C. Fernández y P. Baptista. (2010). Metodología de la Investigación. México: McGraw Hill.

Instituto Nacional de Federalismo (INAFED). (1998). Enciclopedia de los municipios y delegaciones de México, Coatepec. Recuperado de: (http://www.inafed.gob.mx/ work/enciclopedia/EMM30veracruz/municipios/30038a.html)

Katz, D. y Stotland, E. (1959) "A preliminary statement to a theory of attitude structure and change". En Koch, S. (Ed.), Psychology: A Study of a Science: pp. 423-475. MacGraw-Hill, New York.

Knussen, C., Yule, F., MacKenzie, J., y Wells, M. (2004). An analysis of intentions to recycle household waste: The roles of past be- havior, perceived habit and perceived lack of facilities. Journal of Environmental Psychology, 24, 237-246.

LaGrega, M. y Evans, J. (1996). Gestión de Residuos Tóxicos. Tratamiento, eliminación y recuperación de suelos. Mc Graw Hill.

Lizama P., F. (2018). Evaluación de la Agenda para el Desarrollo Municipal como instrumento de planificación sostenible: un estudio de caso en Tabasco, México. Tesis de Doctorado. El Colegio de la Frontera Sur. Villahermosa, Tabasco.

Lizama P., F.; Ortega-Argueta, A.; Sandoval C., M.C.; Piñar-Álvarez, M.A.; Mesa Jurado, M.A y Vivas Rodríguez, J.F. (2016). "Evaluación del Sistema Informático (SIGLO) del Instituto Nacional para el Federalismo y Desarrollo Municipal". En: Torres Guillermo E. [et. al.], Avances y Perspectivas en Computación y Tecnologías de Información y Comunicación. 1ra. Ed. pp.191-211. Tenosique, Tabasco, México: Universidad Juárez Autónoma de Tabasco. Santa Elena, Petén, Guatemala: Universidad de San Carlos de Guatemala.

Malave, N. (2007). Trabajo modelo para enfoques de investigación acción participativa. Programas nacionales de formación. Escala de Likert. Recuperado de: http://uptparia.edu.ve/documentos/F\%C3\%ADsico\%20de\%20Escala\%20Likert.pdf

Mander, J. y E. Goldsmith (ed.) (1996): The case against the global economy: And for a turn toward the local. San Francisco, Sierra Club Books.

Manson, R. H. (2004). Los servicios hidrológicos y la conservación de los bosques de México. Madera y Bosques,10(1).

De Medina S., L. y E. Castillo G. (2014). Políticas públicas municipales en materia de gestión de residuos sólidos. Caso Teocelo. En: García-López, T. (Coord.) Temas selectos de gestión y políticas públicas municipales en materia ambiental. Universidad Veracruzana. Ed. Ubijus. México. pp.125-139.

Nagao, N., Matsuyama, T. y Yamamoto, H. (2003). A novel hybrid system of solid state and submerged fermentation with recyclefor organic solid waste treatment. Process Biochemistry. pp. 1-7 
Piñar-Álvarez, M.Á. (2014). "Agenda desde lo local y políticas públicas municipales en materia ambiental, Veracruz." In: García López, T. Temas selectos de gestión y políticas públicas municipales en materia ambiental, pp. 1-64. Ed. Ubijus Editorial SA de CV. /Universidad Veracruzana. México.

Polit, D.F. y Hungler B. P. (2000). Investigación científica en Ciencias de la Salud: principios y métodos. $6^{\mathrm{a}}$. Ed. México: McGraw-Hill Interamericana.

Rokeach, M. (s/f). Art. sobre" Actitudes", en Enciclopedia de las Ciencias Sociales, t. 1, p. 15.

Sabater, J.M. (1989). Sobre el concepto de actitud. Anales de la Pedagogía. Recuperado de: http://revistas.um.es/analespedagogia/article/viewFile/287671/208941

Sachs, W. y G. Esteva (2003): Des ruines du développement. Paris, Le Serpent à Plumes.

Secretaría de Finanzas y Planeación del Estado de Veracruz (SEFIPLAN) (2015a). Sistema de Información Municipal. Cuadernillos Municipales. Teocelo. SEFIPLAN. México. Recuperado de: http://www.veracruz.gob.mx/finanzas/files/2015/05/Teocelo.pdf

Secretaría de Finanzas y Planeación del Estado de Veracruz (SEFIPLAN) (2015b). Sistema de Información Municipal. Cuadernillos Municipales Coatepec. Recuperado de: http://www.veracruz.gob.mx/finanzas/files/2015/05/Coatepec.pdf

Secretaría de Medio Ambiente y Recursos Naturales (SEMARNAT) (2009). Programa Nacional para la Prevención y Gestión Integral de Residuos 2009-2012. Diario Oficial de la Federación, 2 de octubre de 2009. México.013

Shove, E. (2003). Comfort, Cleanliness and Convenience: the Social Organization of Normality. Berg. ISBN 951-698-120-8

Soumaré, M., Tack, F. y Verloo, M. (2003). Effects of a municipal solid waste compost and mineral fertilizacion on plant growth in two tropical agricultural soils of Mali. Bioresourse Technology. No.86 (2003); p.15-20.

Taylor, S., y Todd, P. (1995). An integrated model of waste manag- ient behavior: A test of household recycling and composting intentions. Environment and Behavior, 27, 603-630.

Troschinetz A.M. y Mihelcic J.R. (2009). Sustainable recycling of municipal solid waste in developing countries. Waste Manage. 29, 915-923.

Williams, G. (2007). El bosque de niebla del centro de Veracruz: ecología, historia y destino en tiempos de fragmentación y de cambio climático. Instituto de Ecología y CONABIO. Recuperado de: https://s3.amazonaws.com/ academia.edu.documents/32412626/bosque_de_niebla.pdf?AWSAccessKeyId=AKIAIWOWYYGZ2Y53UL3A\&Expires $=1504638355 \&$ Signature $=\mathrm{k} 8 \mathrm{biM} 6 \mathrm{tUn}-$ rf0X\%2FWbJRMMY08krFg\%3D\&response-content-disposition=inline \%3B\%20filename\%3DBosque_de_niebla.pdf

Wojtarowski, A., Piñar, A. y Pérez, M. R. (2018). ¿Por qué Teocelo si pudo? Un análisis de los factores de éxito de su programa de gestión integral de residuos sólidos municipales, desde la Teoría del Comportamiento Planificado. Cultura y Representaciones Sociales, 25, 235-278. 